Research Article

\title{
Prediction Model for Information Dissemination in Social Network Media Based on Triangle Ring Attractor
}

\author{
Zhe Zheng $\mathbb{D}^{1},{ }^{1}$ Chunliang Zhou $\mathbb{D},{ }^{2}$ Xiangpei Meng, ${ }^{2}$ Le Wang, ${ }^{2}$ and Ying $\mathrm{Xu}^{2}$ \\ ${ }^{1}$ Ningbo City College of Vocational Technology, Ningbo, China \\ ${ }^{2}$ Ningbo University of Finance \& Economics, Ningbo, China \\ Correspondence should be addressed to Chunliang Zhou; zhouchunliang@nbufe.edu.cn
}

Received 21 June 2019; Revised 29 November 2019; Accepted 28 December 2019; Published 11 February 2020

Academic Editor: Rafal Zdunek

Copyright (c) 2020 Zhe Zheng et al. This is an open access article distributed under the Creative Commons Attribution License, which permits unrestricted use, distribution, and reproduction in any medium, provided the original work is properly cited.

In order to solve the information transfer of social network media, a new information transfer prediction method is presented by a triangle ring attractor. At first, the description method of social media information is given in this model, and the evaluation indexes such as information recall rate, matching degree, and recall rate are emphatically expounded. At the same time, the characteristics of media information are analyzed with a triangular ring attractor, and an information transfer prediction model is established. Finally, through the simulation experiment, the key factors influencing the method are deeply analyzed. Experimental results show that compared with other algorithms, this method has good adaptability in the degree of information attenuation and information checking rate.

\section{Introduction}

With the rapid development of network information technology, Internet-oriented dissemination mode has become the mainstream media in the information era, and has begun to change people's daily life and lifestyle from various aspects. The subjects of information dissemination in social network media are centralized, and the ability to recognize and discriminate the information is affected by the social environment [1-6]. The social network characteristics involved in social media are not analyzed and discussed. These are only surface information, and there is no in-depth analysis of the characteristics of nodes in social networks. Many studies only use social media data as a part of data sources. They are still based on a large number of traditional website data for prediction. Social media information is only predictive factors. Therefore, it is necessary to adopt new methodology or combine a variety of mature theories to predict the impact of information dissemination in social network in this form.

The information dissemination prediction methods of social networks can be generally divided into two categories, namely, macroscopic and microscopic prediction methods.
The research of macropredictive methods mainly starts from the whole of information dissemination and predicts the future propagation situation, spread breadth, propagation depth, speed of propagation, and scope of influence of information dissemination. However, the research on information dissemination at the macrolevel mostly predicts the breadth of information dissemination, the depth of dissemination, and the speed of communication, but ignores the influence of social network topology on information dissemination. Therefore, it is necessary to find indicators further for quantifying the structure of information dissemination structure, so as to accurately establish an information propagation structure morphology prediction model. The research of the microprediction method is mainly based on the details of information dissemination, considering the probability that information will be forwarded again after reaching a certain node. However, most of the microlevel prediction methods are concentrated on the nodes themselves, but the process of information dissemination is usually not independent, and there may be a relationship between competition and cooperation between nodes and nodes. Therefore, it is necessary to consider the competition and cooperation between nodes to establish a 
more accurate information propagation prediction model. For example, the nonnegative matrix decomposition algorithm and the user clustering algorithm are the typical methods for depicting social network media. The nonnegative matrix decomposition algorithm follows the nonnegative constraint condition when decomposing the matrix and uses the gradient descent method to calculate the submatrix, but it is sensitive to outliers in the data set, and it is not easy to identify complex nonlinear data structures. The user clustering algorithm clusters with the text features generated by the user comments, which can optimize the information recommendation, but lacks a deeper representation of the user's point of view.

At present, the main methods to depict and predicate the information dissemination in social network are as follows: Bayesian network model [7], Markov network model [8], relationship dependence network model [9], directed acyclic probabilistic entity relationship model [10], random relationship model [11], etc. And domestic and foreign scholars have proposed a lot of solutions. Shusen et al. [12] elaborated the basic concepts of network, role, etc. in social network; proposed the identification problem of implicit and explicit roles in social network; and analyzed the main methods and research status of these two types of role identification. Yongli et al. [13] established a method to predicate social network links based on decision-making analysis and utility function analysis, which depicted the estimation problem of utility function parameters, and proposed the Markov chain Monte Carlo parameter calibration method to enable users to make reasonable decisions in terms of model efficiency and accuracy. Yingan et al. [14] compared and analyzed multiple sampling methods according to the evolution track of sampling technology development in social media on account of the difficulty of traditional social media sampling method in dealing with its endogenous uncertainty and complexity and tested the above methods using social media data. Xiangwu et al. [15] summarized and analyzed the research status of mobile news recommendation and pointed out the difference between it and traditional news recommendation and other mobile recommendations and then made comparison and analysis on the basis of mobile news recommendation technology of social network, mobile news display, typical applications, etc. Lingling and Junping [16] studied the precise search of cross-media data in the field of emergency management and established a crossmedia information retrieval system, which provided a scientific and accurate decision-making basis for the mining and integration of emergency information. Yezheng et al. [17] selected original posts of the topic and structural data, e.g., number of comments and retweets, etc. based on the statistical characteristics of the topic, and finally got the prediction results of topic hotness by building the hotness curve model. Cataldi et al. [18] took the behavior characteristics of different users and the user relationship into account and judged the topic hotness of microblog through user relationship. Yuzhong et al. [19] proposed a method based on frequent pattern mining of data flow to detect hot topics in microblog flow. The shape-based prediction method considers that the occurrence, development, and change of hot topics show different trends with the change of time, space, and environment, that is, judgement of the trend of topics. Jiuxin et al. [20] discussed the construction and application of classic and popular time-series statistical models. Dakos et al. [21] gave an alarm when reaching key critical point with time-series mining method based on simulated ecological data set. Yanxiang et al. [22] adopted the similarity matrix with invariant scaling and translation to solve the clustering problem of dynamic time series on the basis of the idea of shape clustering. However, the above models have achieved good results in different forecasting fields, but still only use social media as a convenient method to obtain data. The essence is still the traditional event prediction. There are still many problems in the above research that need to be further discussed, and a reasonable information prediction model is constructed by combining the development trend, and the calculation cost is relatively high. Within a defined scope, the network nodes with specific influence are defined as attractors, forming a triangle ring with the other two node clusters with strong and weak relationship, which does not mean that the other two nodes with strong and weak relationship cannot become attractors. When a social member changes, the influence of network structure itself will be affected, which will lead to the reduction of social influence of other two nodes in the ring, and the role of attractors in social network is to drive the structure to move toward the target state, and this traction state is defined as the edge weight of triangle ring, which reflects the influence between nodes.

Therefore, on the basis of the above work, this paper proposes a new prediction model for information dissemination in social media based on a triangle ring attractor [23-25], which firstly presents the depiction method of social media information, analyzes the characteristic quantity of media information based on triangle ring attractor, and establishes the prediction model for information dissemination. Finally, the key factors affecting this method are analyzed deeply through simulation experiments. The structure of this paper is as follows: Section 1 presents the depiction method of social media information; the prediction model for information dissemination is established in Section 2; the simulation experiments are carried out in Section 3; Section 4 summarizes the full text.

\section{Depiction Method of Social Media Information}

Currently, with the rapid development of Internet, social network platform has gradually become the mainstream platform for the communication between people: users expand their own social circle using social network and carry out media entrepreneurship using social media platform, and friends and relatives communicate with each other through social platforms, and entertainments, current affairs, and new things spread rapidly and widely on the Internet media. However, since the trust mechanism of information dissemination itself is not perfect, and the inference algorithm of some computational research models cannot go deep into the information contents, and the 
established trust degree is not convincing, the information safety cannot be guaranteed. In order to predict the safety of information dissemination, this paper will depict the information structure of social network media and establish a distrust screening mechanism based on the uniqueness of information architecture. First of all, it needs to determine the target information network set $u=\left\{u_{1}, u_{2}, u_{3}, \ldots, u_{n}\right\}$, in which $u$ is the information of target user, and social network $N$ and media network $M$ of the user are given. As a result, the social network media of the user is represented as $U=(M, N, u)$. The information received by the user is divided into information $u_{i}$ and effective information $u_{a}, 0 \leq i \leq n$, in which $n$ is the total number of information, and the recall rate $\Psi$ is obtained from the distribution of joint probability $p$ that the user receives effective information:

$$
p\left(u_{1}, u_{2}, u_{3}, \ldots, u_{n}\right)=\prod_{i=1}^{n} p\left[u_{a} \mid p_{n}\left(u_{i}\right)\right], \Psi(U)=\sum_{i=1}^{n}(-1)^{i+1} \cdot\left(\frac{n !}{i !(n-i) !}\right) \cdot 2^{i(n-i)} \cdot p\left(u_{1}, u_{2}, u_{3}, \ldots, u_{n}\right)
$$

The process of information dissemination is refined in this paper. After the information is disseminated to a target user, an error will occur when the target user continues to disseminate the information. Therefore, the attenuation coefficient $\varepsilon$ is derived, which reflects the distortion state in the process of information dissemination, and $Q$ is defined as the average dissemination range of network media $M$ within effective time $t$. In order to search in a wide range of space, this paper proposes a local update formula in combination with reference [26] in view of the difficulty in defining the scope of information dissemination in social network:

$$
Q_{i}^{t+1}=Q_{i}^{t+1} \cdot\left[\varepsilon Q_{i}^{t}+\exp (1-\varepsilon) t\right] .
$$

Assuming that the dissemination scope is set as a multidimensional space, the information is continuously disseminated to the target user who updates the obtained information and then disseminates it to the relevant user. Based on this, a multidimensional network dissemination tree is constructed, and local update and calculation are carried out once during the information dissemination. In a certain time domain, if the information node $w$ is the window period of information dissemination in the social media environment, the information is allocated and disseminated, and the information matching degree $\phi$ is depicted based on the characteristics of node dissemination:

$$
\phi=\sum_{n=1}^{j}\left\{\log \left[\frac{\left(w_{j}-1\right) !}{\left(Q_{n j}+w_{j}-1\right) !}\right]+\sum_{k}^{w_{j}} \log \left(w_{j n k} !\right)\right\} .
$$

The information dissemination in social media network is based on the trust degree, which conforms to uniform distribution. The random variable subject to uniform distribution is set as $\alpha$, and local up-to-date solution $u_{\text {new }}$ is extracted from effective information based on the random walk rule of information dissemination:

$$
u_{\text {new }}=\alpha u_{i}+\left(\frac{u_{i}+\beta Q^{t}}{u_{n}}\right)
$$

where $\beta \in[-1,1]$ is a random natural number. Set $v$ as the dissemination speed in the dissemination scope. Due to different dissemination rates of information network $U$, the recall ratio $\delta$ of effective information in the process of information dissemination is depicted based on the optimal solution:

$$
\delta_{U}=\sum_{i=1}^{n} \alpha u_{i}+\left(\frac{u_{i}+\beta Q^{t}}{u_{n}}\right)+\prod_{i=1}^{n} \frac{\alpha v_{i}^{t}}{v_{n}^{t}} .
$$

So, the objective optimization function of the effective information recall ratio $\delta$ is established by combining the abovementioned parameters such as the dissemination speed $v$, the information matching degree $\phi$, and the average dissemination range $Q$, so as to obtain the optimal information transmission prediction value. The Solution method is given with the triangle ring attractor in the following:

$$
Z(\delta)=\max (\phi, Q, v)
$$

\section{Prediction Method}

However, some hidden or unclear information ("click bait" for short) in real life enters people's vision by forwarding public opinions. In order to effectively exclude such information, identify, and predict the effective information dissemination in social network media, this paper first analyzes the structure of social network and divides the relationship between nodes into information dissemination relationship and receiving relationship according to the dissemination relationship between individuals in the social network [27]. Within a defined scope, the network nodes with specific influence are defined as attractors, forming a triangle ring with the other two node clusters with strong and weak relationship. When a social member changes, for example, a node goes offline (fishing node), the influence of network structure itself will be affected, which will lead to the reduction of social influence of other two nodes in the ring, and the role of attractors in social network is to drive the structure to move toward the target state, and this traction state is defined as the edge weight of triangle ring, which reflects the influence between nodes. Based on the degree of coupling between members, this paper establishes the information dissemination network and proposes an efficient method to calculate the nods with obvious strong and weak relationship.

In order to construct an abstract triangle ring, the process of information dissemination in network media is first depicted, and there are a variety of reasons affecting the information dissemination, and hot topics are easy to arouse people's interest in viewing and discussing information. 
Therefore, SIR model is built to calculate the whole process. Interest-related potential information factor vector $I=\left\{z_{1}\right.$, $\left.z_{2}, z_{3}, \ldots, z_{\mathrm{k}}\right\}$ is introduced, in which $I$ is obtained by counting the items clicked by users, and interest-related potential factors in the social information structure are analyzed. Assuming that the potential factor vector $I$ has $k$ potential factors, local tuple information $\left\langle u, u_{\text {new }}, z>\right.$ is explained, and recommended model SIR in the social network project is established, and $\eta$ function represents the target user and all users who have close relationship with the target user in the social network media, and conditional probability formula of target function $\eta$ is defined:

$$
\chi_{u}[I \mid u, \eta(u)]=\prod_{u_{\text {new }}, \eta(u)} \prod_{i \in I\left(u_{\text {new }}\right)-I(u)} \chi_{u}\left(i \mid u, u_{\text {new }}\right)^{\text {uncertain }}
$$

where $i \in k$, in which $u$ is the user cluster that does not receive the latest information but is easy to receive the information, and $u_{\text {new }}$ is the local user cluster who has received the updated information, and $\chi$ is the probability that the user will disseminate such information. uncertainedis a variable, satisfying the condition

$$
\text { uncertain }= \begin{cases}1, & i \in I\left(u_{\text {new }}\right)-I(u) \\ 0, & \text { otherwise. }\end{cases}
$$

The calculation formula is updated by analyzing the potential factors:

$$
\chi_{u}(I \mid u, \eta(u))=\prod_{u_{\text {new }}, \eta(u)} \prod_{i \in I\left(u_{\text {new }}\right)-I(u)} \sum_{z} \chi(i \mid z) \chi_{u}\left(z \mid u, u_{\text {new }}\right) .
$$

Due to the variability of information dissemination, it is difficult to describe the whole process in words. Therefore, the dissemination multiplier $\gamma$ is derived according to the above indexes, which can reflect the abnormal jitter of information dissemination. Specific derivation process is as follows:

$$
\begin{aligned}
\gamma\left(\chi_{u} ; \sigma\right) & \equiv \gamma\left(\chi_{u} I \mid u, \eta(u) ; \sigma\right) \\
& \Longrightarrow \sum_{u_{\text {new }} \in \eta(u)} \sum_{i \in I\left(u_{\text {new }}\right)-I(u)}\left\{\sum_{z}[\log \chi(i \mid z)]+\log \chi_{u}\left(z \mid u, u_{\text {new }}\right)\right\} \\
& \Longrightarrow \sum_{\left\langle u, u_{\text {new }}, i\right\rangle}\left\{\sum_{z}\left[\log \chi(i \mid z)+\log \chi_{u}\left(z \mid u, u_{\text {new }}\right)\right]\right\}
\end{aligned}
$$

where $\left\langle u, u_{\text {new }}, i\right\rangle: u \times \eta(u) \times I^{\mathrm{rcc}}(u)$ are the data obtained by observation, and $\sigma$ is the parameter to be estimated in SIR model, and the basic formula is as follows:

$$
\sigma=\left[\chi(i \mid z), \chi_{u}\left(z \mid u, u_{\text {new }}\right)\right] .
$$

Secondly, this paper conducts data mining on social network media information, determines the influence of information nodes based on the number of clicks, comments, etc., and identifies the attractors for a specific influence. For example, in the network structure, if $f$ enables surrounding nodes to be disseminated or received one after another, then $f$ can be regarded as an attractor. On the basis of the movement trend of surrounding nodes, a triangular ring based on the influence of the individual is designed to depict the edge weight and attraction degree of the information triangular ring. First of all, two node clusters are located according to the information dissemination process and information reception process, and then the attractors that are disseminated between information nodes are described by attraction degree, and the target node $a\left(K=a_{1}, a_{2}, a_{3}, \ldots, a_{n}\right)$ is determined in the node cluster $K$ of an information tree. Assuming that there is a node $b\left(\zeta=b_{1}, b_{2}, b_{3}, \ldots, b_{m}\right)$ in its adjacent node cluster $\mathrm{N} 1$, the dissemination multiplier $\gamma$ between two nodes is obtained by comparing a large amount of data and deriving the information dissemination process. Therefore, the calculation formula of the edge weight $d$ of the nodes $a$ and $b$ can be obtained as follows based on the edge weight $b$ of information units $a$ and $b$ :

$$
\begin{aligned}
& d_{a, b}=\gamma \sum d(a)_{b, b}^{2}, \\
& d_{a, b}= \begin{cases}1, & b \in \zeta \\
0, & \text { else. }\end{cases}
\end{aligned}
$$

When a member is identified as a fishing node, the stable state of the triangular ring will be broken, and then the attractor will have an effect on the associated information nodes. Therefore, the calculation formula $P_{c}^{\prime}$ of the probability that the ring $p^{\prime}$ tends to be stable according to the impact of edge weight on the ring:

$$
P_{\mathrm{c}}^{\prime} \stackrel{\text { def }}{=} \gamma \cdot \frac{d_{a_{n}} / K}{d_{b_{m}} / W}
$$

The information types, e.g., "click bait," "fishing information," etc., are mapped based on the degree of coupling between information members. After classification, a trust mechanism is established to judge the authenticity of information. At this time, negative information is cofiltered to compare positive information, depict the information attraction degree and reflect the information trust degree. If the attraction degree is positive, then such information is positive information; if the attraction degree is negative or zero, then such information is negative information, that is, false information. Specific depiction process is as 
follows: assuming that $a$ and $b$ are mutually independent, $K$ and $W$ are their respective DOF coefficients, and the calculation formula $F(a, b)$ of attraction degree is derived based on the probability formula of triangle ring in the network and the calculation formula of edge weight:

$$
\begin{aligned}
F(a, b)= & \frac{\Gamma(a+b / 2)}{\Gamma(a / 2) \Gamma(b / 2)}\left(\frac{a}{b}\right)\left(\frac{a}{b} \cdot d_{a, b}\right)^{a / 2-1} \\
& \cdot\left(1+\frac{a}{b} \cdot d_{a, b}\right)^{-(a+b / 2)} /\left[P^{\prime}(K) \cdot P^{\prime}(W)\right] .
\end{aligned}
$$

Since the actual scope of information dissemination is not well defined, the model proposed in this paper will limit the frequency of dissemination. At the same time, in order to effectively calculate the attraction degree within the limited range, the DOF scope of information dissemination is limited as follows by combining the joint probability $p$ of effective information, probability formula of triangular ring, and edge weight calculation formula that triangular ring tends to stable: if $\mu$ is the dissemination limit value, then $\mu$ satisfies

$$
\begin{aligned}
\int F_{p}(a, b) f(p) d p & =\mu, \\
\mu & =1-P^{\prime}\left[\frac{1}{F}>\frac{1}{F_{p}(a, b)}\right] .
\end{aligned}
$$

In the scope of limit value, assuming that the ring $p^{\prime}$ has unit rings $I_{1}$ and $I_{2}$, any arbitrary integer $r$ in the triangle ring $P^{\prime}=T\left(I_{1}, I_{2}, d\right)$ satisfies $r I_{1}-C\left(C \in P^{\prime}\right)$, and $r I$ is reversible in the ring $p^{\prime}$, then the initial value $f$ of ring attractor is calculated in any invertible element $W$ :

$$
f(W)=\mu\left(r I_{1}-C\right) \cdot\left[\sum_{i=0}^{n-2} d\left(r_{1}, r_{2}\right)+\sum_{i=1}^{n-1} d\left(r I_{1}, r I_{2}\right)\right] .
$$

When the information of multiple members is added into the triangle ring $P^{\prime}=T\left(I_{1}, I_{2}, d\right)$, the information $i i(i<l)$ of original members is overlaid to the updated information $j j(j>2)$ of the added member in the information network structure formed during the information dissemination for several times, in which $l$ is the total number of members and $\delta$ is the strength change of a node. The change index $H$ of triangle ring attractor is obtained here based on the strength change of network information nodes:

$$
\begin{aligned}
H= & (2 r-\mu I-1) \sqrt{\left[d\left(I_{1}-I_{2}\right)\right]^{2}} \cdot f \frac{d_{i}+F_{i}}{\sum_{j}\left(d_{j}+F_{j}\right)}\left(\mu_{0}+\delta\right) \\
& +\sum_{j \in \Gamma_{i}} f \frac{d_{j}+F_{j}}{\sum_{l}\left(d_{l}+F_{l}\right)} \delta \frac{\mu_{i j}}{F_{i j}},
\end{aligned}
$$

$\mu_{0}=1$ is set to study the changes of attractors within the time range $t$. When only one member joins, an edge is added during the dissemination. Assuming that the strength of edge weight changes to $2+2 \delta$ for each added edge, then the triangle ring attractor changes to $H^{\prime}$ :

$$
H^{\prime}=2 f(1+\delta) t
$$

The following prediction algorithm of social network media information is established here according to the above methods:

(1) The social network media is constructed to form the information dissemination network structure. In the information network, various parameters of triangle ring attractor are initialized, and the dissemination limit value $\mu^{\prime}$ is set:

$$
\mu^{\prime}=1-P^{\prime}\left[\frac{1}{F}>\frac{1}{F_{p}(a, b)}\right] .
$$

(2) The target triangle ring $P^{\prime}=T\left(I_{1}, I_{2}, d\right)$ is selected in the information network, and information nodes $a$ and $b$ are randomly selected in the ring, and the edge weight of the ring is calculated based on the nodes $a$ and $b$, which reflects the influence of ring attractor. At the same time, the recall rate $\Psi$ of the ring $p^{\prime}$ is calculated based on the edge weight value between the nodes $a$ and $b$ in the process of information dissemination:

$$
\begin{aligned}
P^{\prime} \stackrel{\text { def }}{=} & \gamma \sum d(a)_{b}{ }^{2} \cdot \frac{d_{a_{n}} / I_{1}}{d_{b_{m}} / p\left(I_{1} I_{2}\right)}, \\
\Psi\left(P^{\prime}\right)= & \prod_{i=1}^{n} p^{\prime}\left(u_{a} \mid p_{n}^{\prime}\left(u_{i}\right)\right) \sum_{i=1}^{n}(-1)^{i+1} \\
& \cdot\left(\frac{n !}{i !(n-i) !}\right) \cdot 2^{i(n-i)} \cdot \gamma \sum d(a)_{b}^{2} \\
& \cdot \frac{d_{a_{n}} / I_{1}}{d_{b_{m}} / p^{\prime}\left(I_{1} I_{2}\right)} .
\end{aligned}
$$

(3) The attraction degree $F(a, b)$ between the nodes is obtained using the edge weight of triangle ring $P^{\prime}=$ $T(a, b, d)$ and equation (16), and the attenuation occurring in the process of information dissemination is locally updated and handled based on the attenuation factor $\varepsilon$. At the same time, the information matching degree between random nodes $a$ and $b$ in the triangle ring is calculated as per equation (17): 


$$
\begin{aligned}
F(a, b) & =\left[\frac{(\Gamma(a+b / 2) / \Gamma(a / 2) \Gamma(b / 2))(a / b)\left(a / b \cdot d_{a, b}\right)^{a / 2-1}\left(1+a / b \cdot d_{a, b}\right)^{-a+b / 2}}{\left[P^{\prime}(a) \cdot P^{\prime \prime}(b)\right]}\right], \\
\phi & \left.=\sum_{n=1}^{j}\left[\log \left\{\frac{[(a \longrightarrow b-1)] !}{\left[Q_{n j}+(a \longrightarrow b)-1\right] !}\right\}+\sum_{k}^{\mathrm{ab}} \log (a \longrightarrow b)_{j n k} !\right)\right] .
\end{aligned}
$$

(4) If the information attraction degree is negative or zero, then such information is negative information, that is, false information, and it needs to filter out such information and return to step (2); if the attraction degree is positive, then such information is positive information. The attractor $f$ of the triangle ring $P$ is depicted based on the unidirectional strong and weak relationship existing in the process of information dissemination and the attraction degree, and then the optimal value of recall ratio $\delta$ is calculated based on the change $H$ of attractor (there are similarities and differences between one or more information members) of the triangle ring $P^{\prime}=$ $T(a, b, d)$ in the dissemination process:

$$
\begin{aligned}
f\left(P^{\prime}\right) & =\mu\left(r P^{\prime}-C\right) \cdot F\left[\sum_{i=0}^{n-2} d(a, b)+\sum_{i=1}^{n-1} d(r a, r b)\right] \\
\delta_{p^{\prime}} & =\sum_{i=1}^{H}\left[\frac{\alpha u_{i}+\left(u_{i}+\beta Q^{t}\right)}{u_{n}}\right]+H \prod_{i=1}^{n} \frac{\alpha v_{i}^{t}}{v_{n}^{t}}
\end{aligned}
$$

(5) The results are outputted.

(6) The simulation ends.

\section{Mathematical Simulation}

To effectively verify the algorithm in this paper, this paper discusses and experiments on the authenticity, effectiveness, timeliness, etc. of social media information, and randomly selects and extracts from short messages of social network media as experimental data. Two real social network media, i.e., http://www.sina.com.cn/ and https://www.zhihu.com/ respectively, are selected in this paper as experimental data sources. As a public-oriented social media platform, Sina microblog mainly attracts the people of different ages with deep interest in entertainment exploration. More than 200,000 people post, update, comment, and forward information in real time every day, and users can enhance their connections by following and adding friends, and obviously, there is a strong relationship between the followed users; https://www.zhihu.com/ is a social platform for people to explore knowledge and discuss some scientific topics, and mainly attracts the people with strong desire for knowledge. However, the authenticity of the topics needs to be verified, and there is a certain strong relationship between the users who make a post. Part of the information published by the users with higher reputation is crawled through systematic online crawler technology, including some false information published by marketing accounts, etc., and the data source library is constructed. First of all, the randomly selected experimental data are grouped into 200 pieces of false data (totally fabricated), 200 pieces of "following" data (false information data are added in the dissemination process of real data), and 200 pieces of real and valid data based on the authenticity of information. The data with different authenticity are divided based on the actual situation: the authenticity of false data, "following data," and effective data is " 1 ," " 0 ," and " 1 ," respectively. This algorithm is compared with two typical prediction algorithms in social network information dissemination, nonnegative matrix decomposition algorithm, [28] and the user clustering algorithm [29]. Because the data in the Web is not only large in amount of information, it is generally unstructured. At this time, the stored data matrix has the characteristics of high-dimensional sparseness, and nonnegative matrix decomposition can process and analyze large-scale information and reduce the original data dimension. The user clustering algorithm is simpler and faster. Therefore, this paper compares these two algorithms. The prediction effect of the algorithm in this paper is shown in Table 1.

In order to illustrate the effectiveness of the algorithm, this paper compares it with the common prediction methods of social network media information, as shown in Figure 1, and the user clustering algorithm has poor computing performance for false information, and the recall rate obtained through calculation is less than $50 \%$; the nonnegative matrix decomposition algorithm has low prediction degree and poor prediction performance for real information, so the recall rate is only $65 \%$, while the triangle ring attractor algorithm has good prediction performance for the information with different authenticity, and the recall rate is above 95\%. However, through data mining statistics, the algorithm in this paper has better prediction effect on the information with different authenticity and is close to the real data results compared with nonnegative matrix decomposition algorithm and user clustering algorithm.

In the real situation, the authenticity and effectiveness of social network media information will gradually decrease with the increase of time and dissemination frequency in the dissemination process. Therefore, the attenuation coefficient $\varepsilon$ is derived here to depict the attenuation degree of true and effective information. In order to verify the performance of the algorithm, a specific environment is set here for experiments, which does not satisfy the rule that the longer the time is in real environment, the greater the attenuation coefficient, and the attenuation coefficient is randomly assigned by the computer. According to the experimental results as shown in Figure 2, with the increase of time, the 
TABLE 1: Comparison of experimental results.

\begin{tabular}{lccc}
\hline Authenticity & $\begin{array}{c}\text { Algorithm adopted } \\
\text { Triangle ring } \\
\text { attractor }\end{array}$ & $\begin{array}{c}\text { Nonnegative matrix } \\
\text { decomposition }\end{array}$ & $\begin{array}{c}\text { User } \\
\text { clustering }\end{array}$ \\
\hline 1 & 198 & 180 & 175 \\
0 & 196 & 170 & 160 \\
-1 & 199 & 150 & 100 \\
Total & 593 & 500 & 435 \\
\hline
\end{tabular}

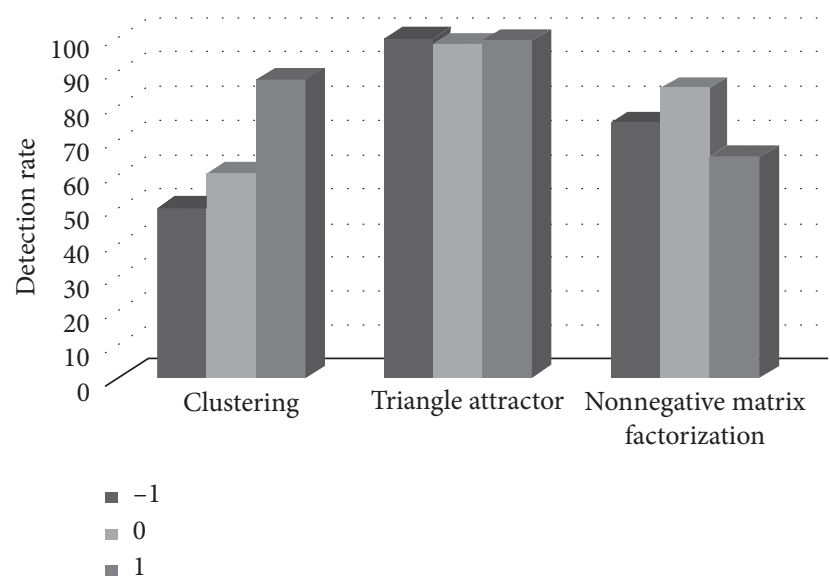

FIGURE 1: Schematic diagram of recall rate $\Psi$ of different algorithms based on information authenticity.

attenuation coefficient in the nonnegative matrix decomposition algorithm starts to exceed 10 in the second day and then increases continuously, and the attenuation coefficient in the user clustering algorithm starts to exceed 10 in the fifth day, which exceeds the nonnegative matrix decomposition algorithm. It can be seen that the user clustering algorithm has low adaptability, and the attenuation coefficient of triangle ring attractor does not exceed 10 after 45 days. It thus can be seen that the algorithm in this paper can preserve the information well.

In general, under the influence of user's subjective emotion, the authenticity of negative information will be affected by pessimism and misery indexes in the dissemination process, and the information containing negative information is marked as negative information, which thus affects the judgement on information authenticity, and the recall ratios of three algorithms are calculated and compared under different negative information conditions by properly cofiltering the negative information, i.e., under the action of triangle ring attractor. As shown in Figure 3, the influence factor is set as negative information, and the negative information is disseminated. The results show that the effective recall ratio of nonnegative matrix decomposition algorithm is greatly affected by negative information, and the recall ratio decreases as the proportion of negative emotion in subjective information increases, and the user clustering algorithm has better adaptability to the influence of negative information, but the recall ratio still does not exceed $80 \%$, which is inferior to the impact of triangle ring attractor on the information. It can be seen from the comparison that the

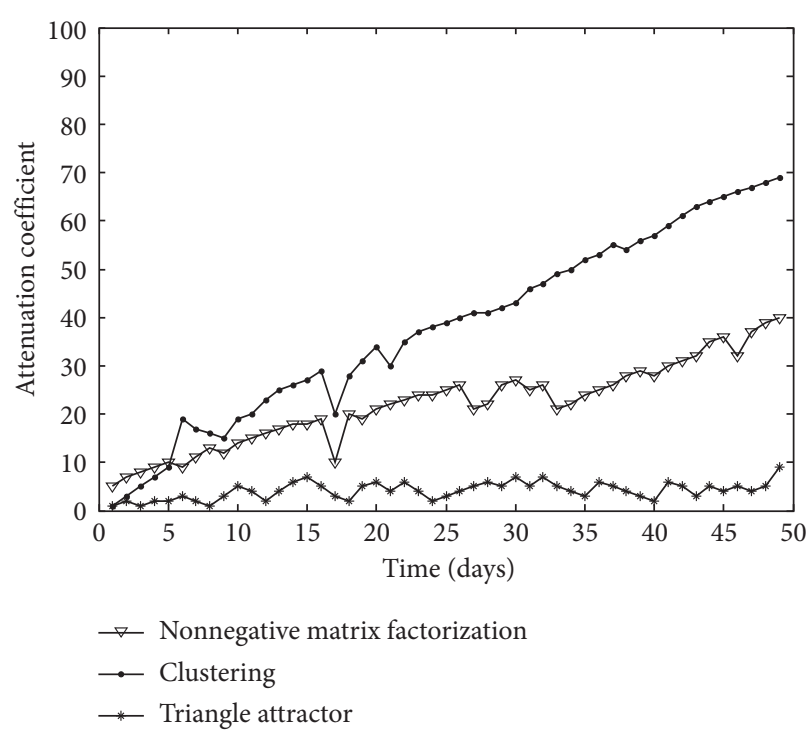

FIGURE 2: Comparison of information attenuation degreesof different algorithms at different times.

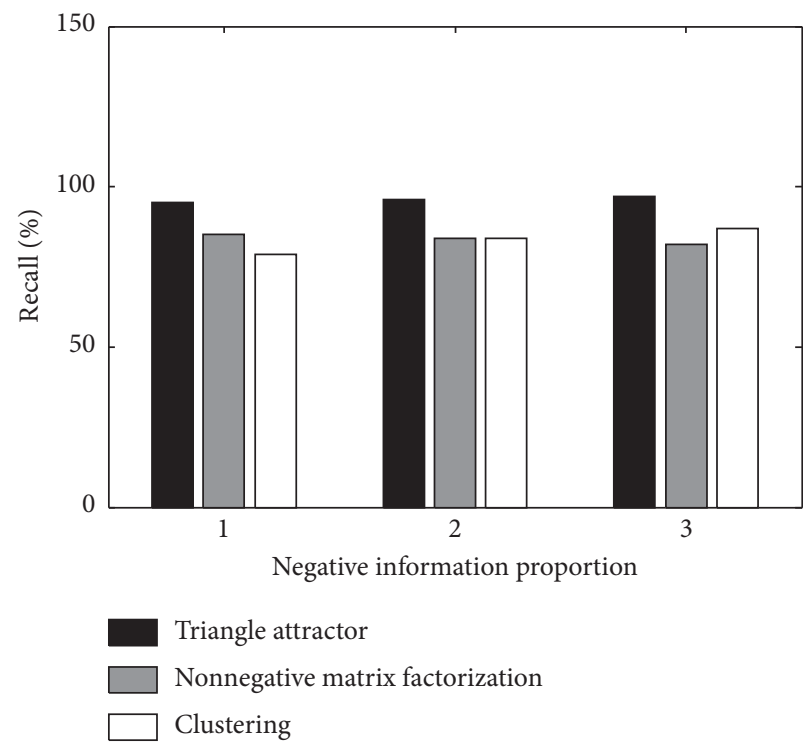

FIGURE 3: Recall ratio $\delta$ of negative information influence under different algorithms.

algorithm in this paper has good adaptability to the negative information, and the recall ratio is not lower than $95 \%$.

At the same time, the information matching degree $\phi$ of the three algorithms is compared in Figure 4. It can be seen from Figure 4 that during the experimental period, the information matching degree of the triangle ring attractor algorithm is much higher than that of the other two algorithms. This is because the nonnegative matrix decomposition algorithm uses the gradient descent method to calculate it, and it is sensitive to outliers in the data set and does not easily identify complex nonlinear data structures. But for the user clustering algorithm, When the density changes, the distance threshold varies with the cluster, and 


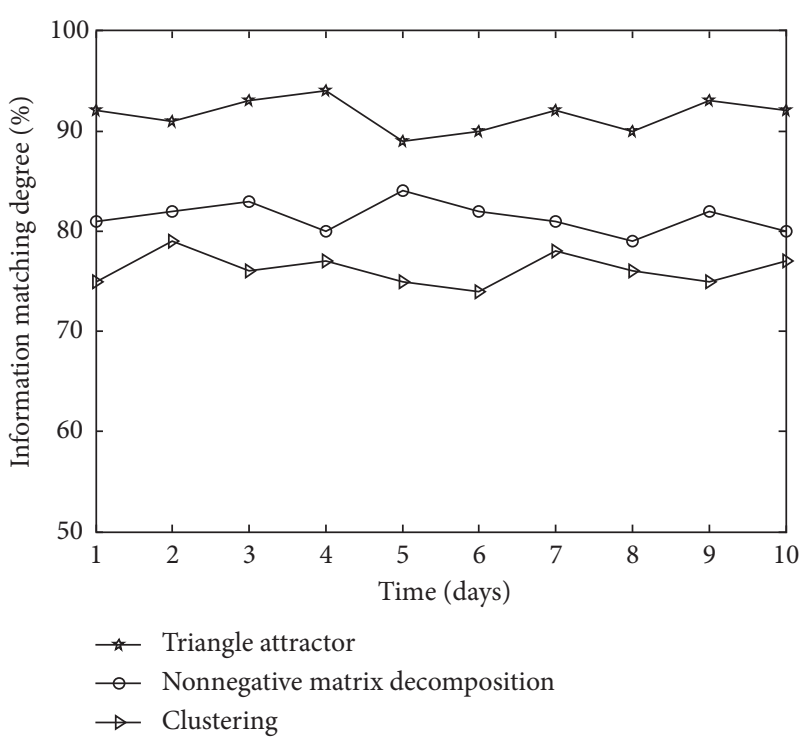

FIgURE 4: Comparison of information matching degree.

the high-dimensional data become difficult to estimate, which reduces the information matching degree.

In addition, it can be seen from the experimental calculation that the attraction degree has an impact on the recall ratio of calculation results, as shown in Figure 5. In this paper, the recall ratio reflects the depth and breadth of information citation. With the change of attractors between information network nodes, the recall ratios obtained by these three algorithms have increased, but compared with common algorithms, e.g., user clustering algorithm and nonnegative matrix decomposition algorithm, even under the condition that the attraction degree is lower, the recall ratio of triangular ring attractor algorithm is more than $85 \%$, and the increased value has been higher than other two algorithms. It can be seen that better prediction effect can be achieved by affecting the nodes in the ring with attractors and correcting the predication model.

At the same time, according to the effective experimental statistics, the time effect of information after 30 days is generally close to 0 , so the time effect within 30 days is considered here when calculating the edge weight. In this paper, in order to reflect the role of triangle ring attractor, the statistical data are calculated under a certain time condition to get the schematic diagram as shown in Figure 6. When disseminating the information, the edge weight value is not 0 , which indicates that the false information is mixed in it at the beginning, and with the increase of time, the edge weight value increases significantly, and the false information disseminated by the information increases significantly and reaches a peak on the $15^{\text {th }}$ day, which indicates that people intensively discuss the topics from the $1^{\text {st }}$ day to the $15^{\text {th }}$ day, resulting in the largest increase of false information in the process; from the $15^{\text {th }}$ day to the $30^{\text {th }}$ day, the hotness decreases, and the edge weight value tends to decline, which is also affected by other factors, e.g. reduction of marketing effort by marketing account, etc. The simulation calculation is also conducted on dissemination multiplier $\gamma$ in this

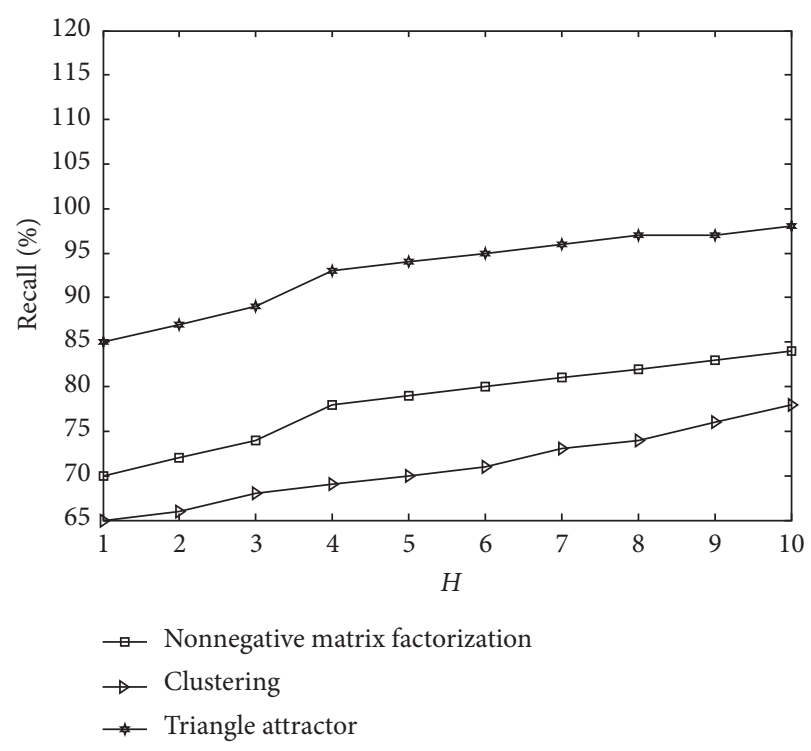

FIgURE 5: Comparison of information recall ratio $\delta$ under different attraction degree changes.

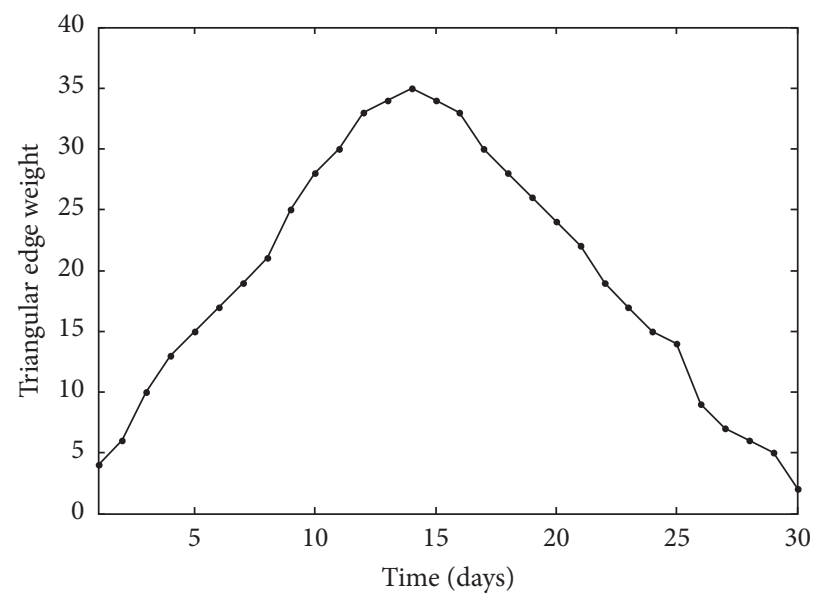

FIGURE 6: Changes of attractor influence at different times.

paper, as shown in Figure 7, and the dissemination multiplier reflects the abnormal jitter of information dissemination. For example, for some repeated sending and dissemination behaviors, $\gamma$ changes in approximately negative proportion within 30 days, and there is jitter on the $15^{\text {th }}$ day, which means the $15^{\text {th }}$ day is a transitional stage and is the feedback of the role of triangle ring attractor.

In addition, this paper also compares the index changes under multiple condition factors. The information dissemination multiplier $\gamma$ is proposed based on the SIR model above to verify the relationship between the multiplier and edge weight of information triangle ring, and this paper compares the calculation results through simulation experiments. Firstly, the environment variables are changed, and the attraction effect has a certain timeliness in the process of information dissemination, so only the time (days) of information dissemination is guaranteed to be different in the process of discussion to achieve the optimal 


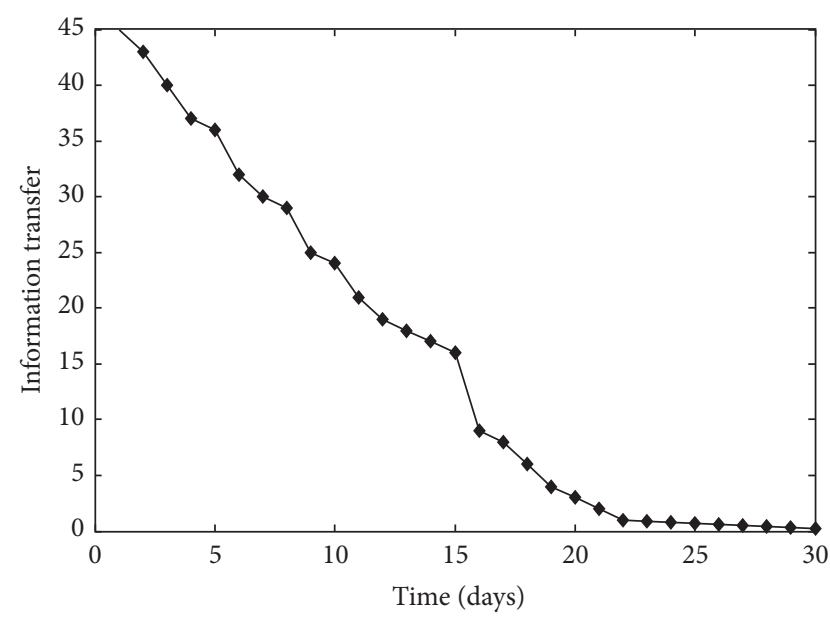

FIGURE 7: Changes of dissemination multiplier $\gamma$ at different times.

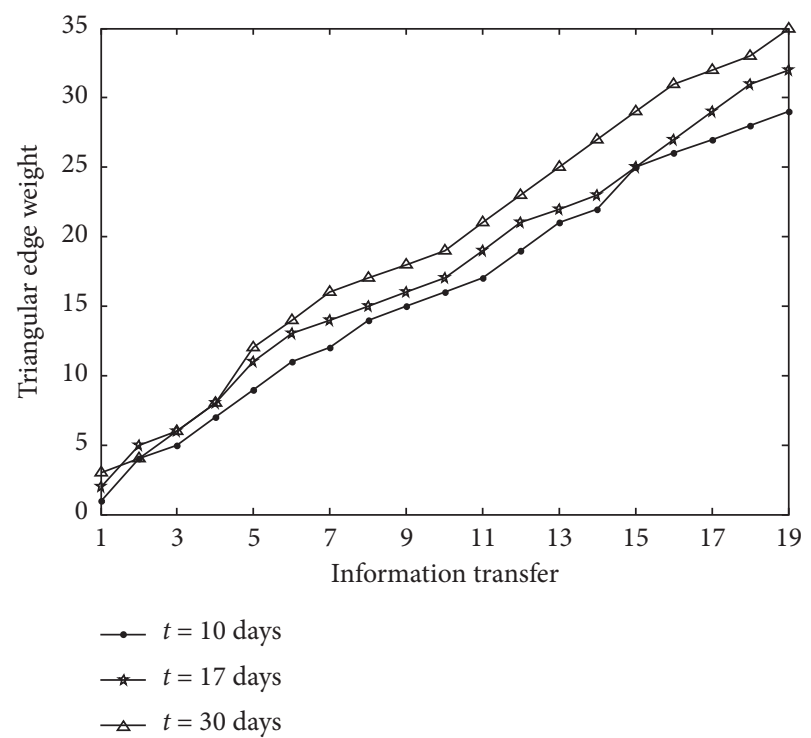

FIGURE 8: Changes of edge weight $\mathrm{d}$ of triangle ring under different dissemination multipliers $\gamma$.

influence effect. The simulation results are shown in Figure 8 , and the data within 10 days, 17 days, and 30 days are captured for comparison. It can be seen from the span between curve lines that the influence of attractor is more significant and presents an increasing trend in direct proportion with the increase of dissemination time on the platform. In a certain period of time, the larger the information dissemination multiplier is, the more obvious the influence of triangle ring attractor on the whole ring is and the more obvious the side reaction ring fluctuation is, which indirectly reflects that the ring fluctuates significantly, and the effect that attractors guide to keep stable is obvious. The longer the information ferments with time, the greater the distortion, and the more obvious the span deviates from the essence of the matter, which is consistent with the effect of weight increase.

This paper depicts the influence of triangle ring attractor using edge weight value, and different edge weight values are

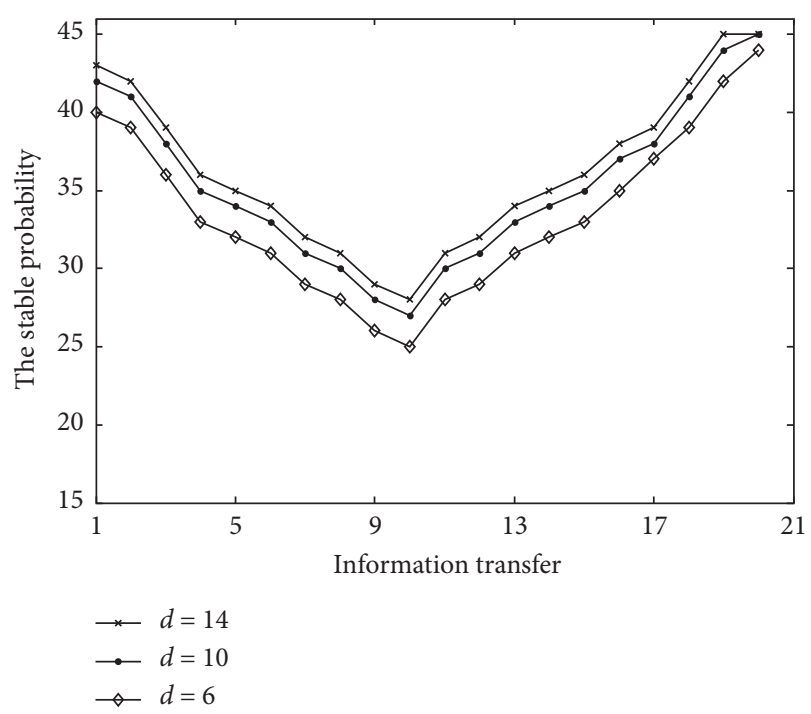

FIGURE 9: Influence of different multipliers $\gamma$ and edge weight $\mathrm{d}$ on $P_{c}^{\prime}$.

provided here to calculate the probability that the triangle ring tends to be stable. Generally, the triangular ring tends to be stable under the action of edge weight. Considering that there are many kinds of incentives affecting the edge weight, this paper focuses on the comparison of the changes in information dissemination multiplier, and the time period is planned from the $6^{\text {th }}$ day to the $15^{\text {th }}$ day. The experimental results are shown in Figure 9. As the edge weight value increases, it can be seen from the broken line distance that $P_{c}^{\prime}$ also increases; however, when observing a broken line, the reason for the decrease of $P_{c}^{\prime}$ value is that the number of attractors changes. Even if the information multiplier increases, the attractors will also be limited by $\mu$ in the real situation. Therefore, the attractors are depicted.

There are many factors affecting the attractor, and one of the most significant factors is the dissemination limit, which reflects the upper limit of dissemination frequency to control the defect that the dissemination scope is wide. The relationship between $\mu$ and $f$ is compared here, and during the 30-day experimental period, as shown in Figure 10, attractors are significantly affected by $\mu$ in $1-13$ days; $\mu$ has almost no effect in 14-30 days. This is because that with the increase of dissemination time, the hotness increases first and then decreases, and the corresponding scope and frequency of dissemination change first along the trajectory. When false information is mixed in it, the number of attractors increases with the change of $\mu$ to maintain a steady state, but when the hotness decreases, the whole information network tends to be stable, and the demand for attractors no longer increases. In general, the dissemination limit only plays a role in the peak period.

To depict the information from various aspects, this paper also compares the correlation between $\mu$ and $\mathrm{F}$. Similarly, 30-day dissemination time is set, and $\mu=30$, $\mu=20$, and $\mu=10$ are also set to capture the data under three conditions for calculation, as shown in Figure 11. The results show that the attraction degree increases with time on 


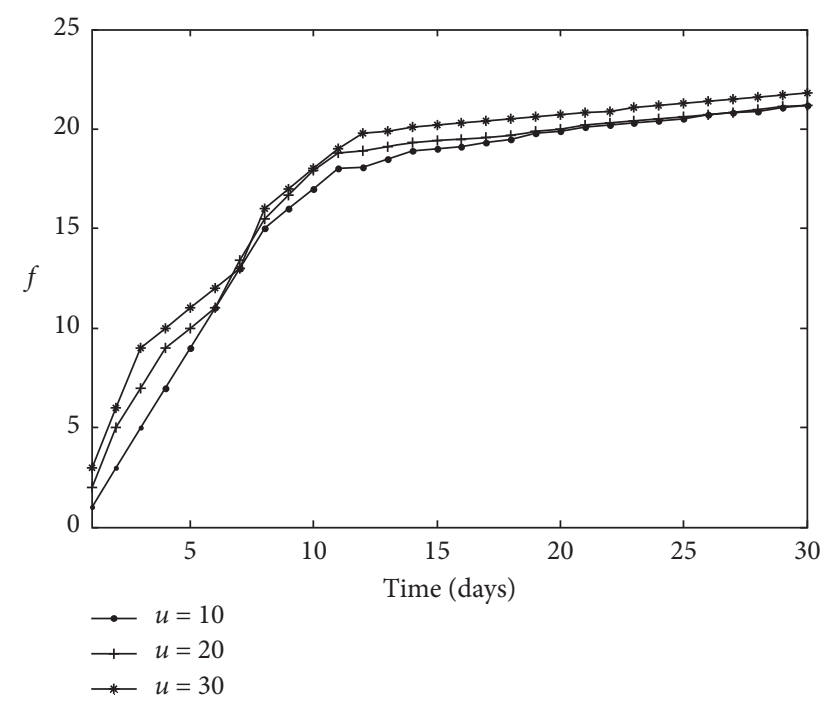

Figure 10: Changes of $f$ attractor at different time and dissemination limit $\mu$.

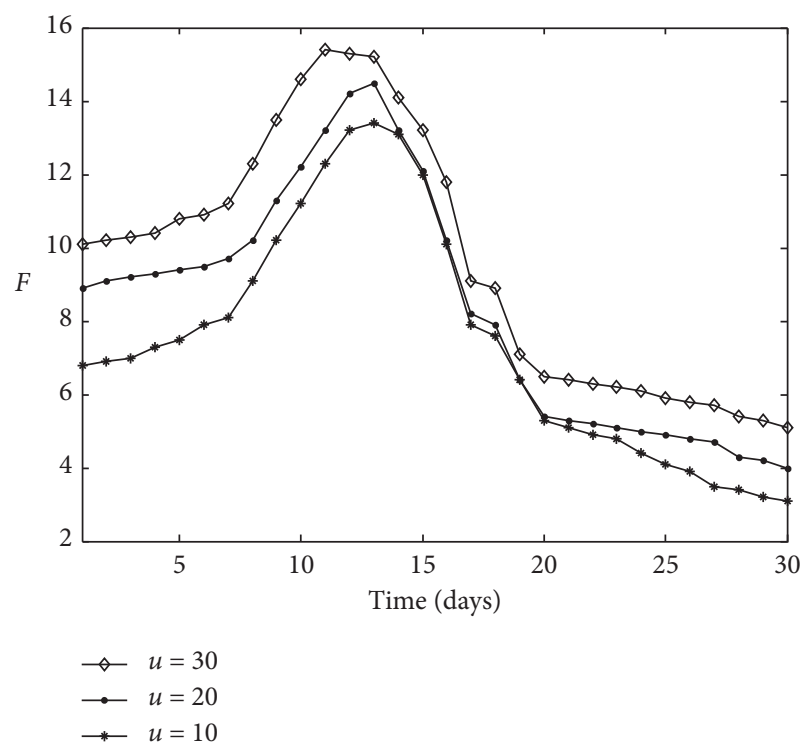

Figure 11: Changes of $\mathrm{F}$ attractor at different times and dissemination limit $\mu$.

the thirteenth day, and then decreases sharply after the hotness gradually decreases. On the eighteenth day, the attraction degree decreases slowly but still decreases. It can be speculated that users no longer pay attention to this information at this time, and some follow-up information is not forwarded. Moreover, the dissemination multiplier also limits the changes of attraction degree, which indicates that the dissemination frequency also has an impact on the attraction degree, and the influence peaks in 1-15 days and 20-30 days.

\section{Conclusions}

In order to effectively solve the dissemination problem of social network media information, this paper proposes a new prediction model for information dissemination based on triangle ring attractor, which firstly presents the depiction method of social media information, and focuses on the evaluation indexes of information recall rate, matching degree and recall ratio, and analyzes the characteristic quantity of media information based on triangle ring attractor, and establishes the prediction model for information dissemination. Finally, the key factors affecting this method are analyzed deeply through simulation experiments. Experimental results show that this method has better adaptability in terms of information attenuation degree and information recall ratio compared with other algorithms. Fractal characteristics of social network media information can be considered in the follow-up study, and a variety of artificial intelligence methods can be combined to improve the prediction model for social network media information.

\section{Data Availability}

The data used to support the findings of this study are available from the corresponding author upon request.

\section{Conflicts of Interest}

The authors declare that they have no conflicts of interest.

\section{Acknowledgments}

This paper was supported by Zhejiang Provincial Natural Science Foundation of China (LY20G030025), National Social Science Foundation of China (18BGL184), and Ningbo Natural Science Foundation (No. 2018A610200).

\section{References}

[1] J. Fagnan, R. Rabbany, M. Takaffoli, E. Verbeek, and O. R. Zaïane, "Community dynamics: event and role analysis in social network analysis," Advanced Data Mining and Applications, vol. 10, pp. 85-97, 2014.

[2] A. J. T. Lee, F.-C. Yang, H.-C. Tsai, and Y.-Y. Lai, "Discovering content-based behavioral roles in social networks," Decision Support Systems, vol. 59, pp. 250-261, 2014.

[3] I. Jahnke, "Dynamics of social roles in a knowledge management community," Computers in Human Behavior, vol. 26, no. 4, pp. 533-546, 2010.

[4] K. Song, D. Wang, S. Feng, and G. Yu, "Detecting opinion leader dynamically in chinese news comments," Lecture Notes in Computer Science, vol. 10, pp. 197-209, 2011.

[5] W. Yuxiang, Q. Xiuquan, L. Xiaofeng, and M. Luoming, "Research on context-awareness mobile SNS service selection mechanism," Chinese Journal of Computers, vol. 15, pp. 2126-2135, 2010.

[6] M. Zheming, "The research on the mechanism of social network media information transmission and translation," Information Science, vol. 24, pp. 8-32, 2017.

[7] J. Sandra, A. Eva, A. Kathleen, and H. Grant, "From science to management: using Bayesian networks to learn about Lyngbya," Statistical Science, vol. 45, pp. 36-41, 2014.

[8] F. Schlüter, F. Bromberg, and A. Edera, "The IBMAP approach for Markov network structure learning," Annals of 
mathematics and artificial intelligence, vol. 23, pp. 197-223, 2014.

[9] T. Khot, S. Natarajan, K. Kersting, and J. Shavlik, "Gradientbased boosting for statistical relational learning: the relational dependency network case," Machine Learning, vol. 56, pp. 25-56, 2012.

[10] L. Yan and Z. M. Ma, "Incorporating fuzzy information into the formal mapping from web data model to extended entityrelationship model," Integrated Computer-Aided Engineering, vol. 19, no. 4, pp. 313-330, 2012.

[11] S. O'Rourke, "Gaussian fluctuations of eigenvalues in Wigner random matrices," Journal of Statistical Physics, vol. 138, pp. 1045-1066, 2010.

[12] Z. Shusen, L. Xun, and Q. Jinshan, "A review on role identification methods in social network," Chinese Journal of Computers, vol. 30, pp. 649-673, 2017.

[13] L. Yongli, L. Peng, and Z. Shurui, "Link prediction in social networks based on decision analysis," Journal of Management Sciences in China, vol. 22, pp. 64-74, 2017.

[14] C. Yingan, L. Xue, W. Zhixiao, and Z. Deyun, "A comparison on methodologies of sampling online social media," Chinese Journal of Computers, vol. 54, pp. 1859-1876, 2014.

[15] M. Xiangwu, C. Cheng, and Z. Yujie, "A survey of mobile news recommend techniques and applications," Chinese Journal of Computers, vol. 127, pp. 685-703, 2016.

[16] Z. Lingling and D. Junping, "Study on cross media information retrieval system based on emergency," Computer Simulation, vol. 172, pp. 16-19, 2012.

[17] L. Yezheng, D. Yanan, J. Yuanchun, and D. Fei, "Trend prediction for microblog based on classification modeling of heat curves," Pattern Recognition and Artificial Intelligence, vol. 154, pp. 27-34, 2015.

[18] M. Cataldi, L. D. Caro, and C. Schifanella, "Personalized emerging topic detection based on a term aging model," ACM Transactions on Intelligent Systems and Technology, vol. 5, no. 1, pp. 1-27, 2013.

[19] C. Yuzhong, F. Mingyue, G. Wenzhong, and G. Kun, “Topic popularity prediction of microblog based on wavelet transformation and ARIMA," Pattern Recognition and Artificial Intelligence, vol. 27, pp. 586-584, 2015.

[20] C. Jiuxin, W. Jiangliu, S. Wei, L. Bo, Z. Xiao, and L. Junzhou, "Sina microblog information diffusion analsis and prediction," Chinese Journal of Computers, vol. 145, pp. 780-789, 2014.

[21] V. Dakos, S. R. Carpenter, W. A. Brock et al., "Methods for detering early warning of critical transitions in time series illustrated using simulated eeological data," PLoS One, vol. 8, 2012.

[22] H. Yanxiang, L. Jianbo, L. Nan, P. Min, C. Qiang, and H. Jing, "Based on improved malthusian model microblogging topic trend forecast," Journal on Communications, vol. 189, pp. 1-8, 2014.

[23] Z. Guangchao, L. Chongxin, and W. Yan, "Dynamic analysis and finite time synchronization of afractional-order chaotic system with hidden attractors," Acta Physica Sinica, vol. 234, 2018.

[24] X. Haiying, Z. Cheng, C. Fengtian, and Z. Wei, "Network selection algorithm based on cell attractor selection scheme," Journal of Beijing University of Aeronautics and Astronautics, vol. 354, pp. 711-717, 2016.

[25] M. Xuezhi, X. Yong, L. Jianping, and M. Huiquan, "Grid chaotic attractors based on arc tangent and its secure communication," Journal on Communications, vol. 276, pp. 106115,2014
[26] H. Yi, X. Jin, F. Binxing, Z. Bin, and J. Yan, "Structural supportiveness theory on social networks," Chinese Journal of Computers, vol. 35, pp. 905-915, 2014.

[27] G. Petz, M. Karpowicz, H. Fürschuß, A. Auinger, V. Stříteský, and A. Holzinger, "Opinion mining on the web 2.0-characteristics of user generated content and their impacts," Human-Computer Interaction and Knowledge Discovery in Complex, Unstructured, Big Data, vol. 445, pp. 35-46, 2013.

[28] Y. Feng, J. Xiao, K. Zhou, and Y. T. Zhuang, “A locally weighted sparse graph regularized non-negative matrix factorization method," Neurocomputing, vol. 879, pp. 68-76, 2015.

[29] W. Zhang, M. Xu, and Q. Jiang, "Opinion mining and sentiment analysis in social media: challenges and applications," HCI in Business, Government, and Organizations, vol. 10923, pp. 536-548, 2018. 